Published in J. Vac. Sci. Technol. B 23, 1174 (2005).

\title{
Recent Developments in Surface Studies of GaN and AIN
}

\author{
R. M. Feenstra ${ }^{*}$, Y. Dong and C. D. Lee ${ }^{\S}$ \\ Dept. Physics, Carnegie Mellon University, Pittsburgh, PA 15213 \\ J. E. Northrup \\ Palo Alto Research Center, 3333 Coyote Hill Road, Palo Alto, CA 94304
}

\begin{abstract}
Recent developments in the structural analysis of GaN and AlN surfaces are reviewed, and the implications of these structures for molecular beam epitaxial growth are discussed. The $\mathrm{GaN}(0001)$, $\mathrm{AlN}(0001)$, and $\mathrm{GaN}(10 \overline{1} 0)$ surfaces are all found to be terminated by metallic layers containing approximately one bilayer of $\mathrm{Ga}$ or $\mathrm{Al}$ atoms. However, in contrast to $\mathrm{GaN}(0001)$ where the Ga-bilayer exists in an incommensurate, fluid-like state at room temperature, the metallic layers for $\mathrm{AlN}(0001)$ and $\mathrm{GaN}(10 \overline{1} 0)$ form large-unit-cell commensurate structures with static atomic arrangements. Small amounts of $\mathrm{H}$ on the $\mathrm{GaN}(0001)$ surface leads to facet formation on the surface, whereas larger amounts of $\mathrm{H}$ produce a new $2 \times 2$ surface arrangement that displaces the Gabilayer. A possible model for the H-terminated $\mathrm{GaN}(10 \overline{1} 1)$ surface is introduced and first-principles total energy calculations employing a finite temperature thermodynamics approach are employed to determine the conditions in which it could be stable.
\end{abstract}

\section{Introduction}

Considerable effort over the past decade has gone into the epitaxial growth of GaN because of its applications for both blue-to-UV optical devices and high power microwave devices. For molecular beam epitaxy (MBE), an understanding of the surface structures during growth promises to provide better control of the growth and the subsequent heterostructures. In particular for plasma-assisted MBE (PA-MBE), in which the source $\mathrm{N}_{2}$ gas is introduced through an RF-plasma source, considerable progress has been made over the past 8 years in determining the relevant surface structures (see Feenstra et al. for a review) ${ }^{1}$.

When grown by PA-MBE under Ga-rich conditions, the $\mathrm{GaN}(0001)$ surface is known to be terminated by slightly more than a bilayer of Ga atoms existing in an incommensurate metallic state. ${ }^{2,3,4}$ At room temperature these Ga atoms, or at least those in the top layer of the bilayer, are in a fluid-like state exhibiting fast $(<\mathrm{ms}$ time scale) atomic jumps. The electron diffraction pattern at room temperature exhibits a six-fold ring of intensity maxima around each primary spot, with two different surface structures occurring in which the separation between the primary spots and the subsidiary maxima are either about $1 / 6$ or $1 / 12$ of the primary spacing. ${ }^{2,5}$ These surface structures were denoted by Smith et al. as "1×1" (including the quotation marks), or pseudo- $1 \times 1$, and it was proposed that the Ga-Ga spacing in the bilayer is approximately $2.8 \AA$ corresponding to that of bulk Ga, as opposed to the $3.2 \AA$ spacing of GaN. ${ }^{2}$ Thus, approximately 7 unit cells of the $\mathrm{Ga}$ fit onto 6 unit cells of the $\mathrm{GaN}$ in an incommensurate arrangement,

\footnotetext{
*feenstra@cmu.edu

$\S$ present address: Raytheon RF Components, 362 Lowell St, Andover, MA 01810
} 
thereby forming a structure consistent with the one seen in diffraction having the observed 1/6 spacing. (No detailed difference between the structures for the diffraction patterns with $1 / 6$ and 1/12 spacing has been determined to date). At temperature above about $200^{\circ} \mathrm{C}$ the subsidiary maxima in the $" 1 \times 1 "$ structure disappear and the pattern becomes simply $1 \times 1$, indicating a completely disordered arrangement of the Ga bilayer on the surface.

First-principles theoretical work has been performed on the " $1 \times 1 "$ structure as well as on a variety of surface structures observed for less Ga-rich conditions. ${ }^{6,7}$ The incommensurate structure can be described as a laterally contracted bilayer (LCB) structure. $^{7}$ In one such LCB model the top layer of Ga contains $4 / 3$ of a monolayer (1 monolayer $=\mathrm{ML}=1.14 \times 10^{15}$ atoms $/ \mathrm{cm}^{2}$ for the (0001) surface), with the second layer containing a single monolayer with the Ga atoms directly on top of the underlying $\mathrm{Ga}$ atoms. A structure with $4 / 3$ of a monolayer implies a lateral contraction of the in-plane Ga-Ga spacing from a bulk spacing of $a$ to $0.866 a \sim 2.75 \AA$. This is very close to what one expects for an optimum spacing based on what is known about bulk Ga and also based on calculations for the energy of free-standing hexagonal Ga bilayers. ${ }^{2}$ For this reason the formation energy of the structure with 4/3 ML is expected to be an excellent approximation to that of the optimal (incommensurate) structure. Calculations for a 4/3 ML structure can be performed using a $\sqrt{3} \times \sqrt{ } 3-\mathrm{R} 30^{\circ}$ cell. (The use of a $\sqrt{3} \times \sqrt{3}-\mathrm{R} 30^{\circ}$, for computational efficiency, implies an orientation of the top $\mathrm{Ga}$ layer rotated by $30^{\circ}$ relative to the underlying layer, but there is no reason to expect that this is the optimum orientation.) The corrugation energy of the top layer with respect to the underlying layer is very small, less than $10 \mathrm{meV} /$ atom. ${ }^{7}$ This $4 / 3$ monolayer LCB structure is found to be the minimum energy surface structure under Ga-rich conditions. A recent analysis of low-energy electron diffraction (LEED) intensity data provides evidence that the second Ga layer is indeed bonded directly on top the underlying GaN layer ${ }^{8}$ (although it should be noted that the subsidiary diffraction maxima associated with the top Ga layer were not observed in that study).

Regarding the relevance of the Ga-bilayer structure to GaN growth by MBE, it is well known for PA-MBE that the presence of the Ga bilayer leads to a smooth growth morphology whereas growth under $\mathrm{N}$-rich conditions produces a rough morphology. ${ }^{9,10,11}$ This difference between the Ga-rich and N-rich morphologies indicates a higher surface diffusivity for the former case, which is interpreted as arising from subsurface diffusion of the active $\mathrm{N}$ species on the surface within a metal layer on the surface. ${ }^{12}$ This mechanism was demonstrated theoretically for In-terminated GaN surfaces but is also believed to occur for Ga-terminated surfaces; it is found that $\mathrm{N}$ is unstable on the surface of the metallic bilayer, i.e. it moves to a subsurface site with no barrier for this motion, and that the diffusion barrier for subsurface diffusion is significantly reduced compared to diffusion on a surface lacking the metallic bilayer. ${ }^{12}$

Growth under N-rich conditions, without the presence of the Ga-bilayer, yields a relatively rough surface morphology since the diffusion barriers in that case are correspondingly large. It is important to note that simply the use of higher growth temperatures in MBE cannot be used to overcome this limitation, since GaN decomposes in vacuum at temperatures above about $800^{\circ} \mathrm{C}$. Rather, use of the Ga-bilayer to enhance diffusivity (the so-called "autosurfactant" effect of the bilayer) ${ }^{12,13}$, or use of some other surfactant layer, is necessary in order to obtain step-flow growth and flat morphology. 
Although the Ga bilayer on the surface is beneficial in terms of surface morphology, it does have some detrimental effects. First, for the rather high Ga fluxes needed to maintain a full bilayer coverage on the surface $\mathrm{Ga}$ droplets are found to form, and if growth is performed under less Ga-rich conditions (such that the droplets do not form) then it is found that portions of the surface form facetted areas. ${ }^{9,10,11,14}$ The nucleation of these facets could possibly be influenced by dislocations intersecting the surface, but it is likely that subsequent growth of the facet faces occurs not because of the dislocations but because the facets are low-energy surfaces. Also, most probably the facets are not terminated by a bilayer-type Ga structure (since they form when the Ga flux is restricted). It is not clear at present whether or not a nonzero-sized growth window exists where the morphology can be maintained as totally flat (i.e. without facets) and also without droplets, although this questions is the topic of ongoing research. ${ }^{14}$ Another deleterious effect of the Ga-bilayer is that it causes $\mathrm{Ga}$ incorporation in the cores of threading dislocations that have full or partial screw character, and these Ga-filled cores are found to produce leakage currents. ${ }^{15,16}$ Theoretically, such Ga-filled cores are found to be energetically favorable. ${ }^{17}$ Moreover, the calculations indicated that such cores would be metallic.

In contrast to the above results for PA-MBE, it appears likely that for reactive MBE (RMBE) using ammonia as a source of nitrogen that the Ga-bilayer is not present during growth. In this case growth is performed under $\mathrm{N}$-rich conditions and a $2 \times 2$ structure is observed on the surface. ${ }^{18,19,20}$ Theoretically a variety of $2 \times 2$ structures involving $\mathrm{H}, \mathrm{NH}_{2}$ or $\mathrm{NH}_{3}$ termination of the surface have been predicted under similar conditions of temperature and $\mathrm{H}$ pressure, ${ }^{21}$ and thus the $2 \times 2$ structure seen in RMBE likely is associated with one of these structures. It is notable that relatively smooth growth morphology can be obtained during RMBE growth, at temperatures similar to that used for PA-MBE i.e. about $750^{\circ} \mathrm{C}$. This observation suggests that the $\mathrm{H}$ or ammonia termination of the surface is effective at reducing the surface diffusion barriers, i.e. acting as a surfactant. Other surfactant layers have also been investigated on the $\mathrm{GaN}(0001)$ surface, including In, ${ }^{12,22,23} \mathrm{Mg},{ }^{24,25}$ and possibly $\mathrm{As}^{20,26}$ (see Neugebauer et al. for a review of surfactants and anti-surfactants on $\mathrm{GaN}(0001)$ surfaces $)^{27}$.

\section{II. $\operatorname{AIN}(0001)$}

The band gap of AlN is significantly higher than that of $\mathrm{GaN}$ (6.3 vs. $3.5 \mathrm{eV}$ ), and thus AIN serves an important role for UV optical devices and for barrier or isolation layers in electrical devices. It is also notable that the bond strength of AIN is significantly higher than that of $\mathrm{GaN}$ (cohesive energies of 2.88 vs. $2.24 \mathrm{eV} /$ bond, respectively) and we thus can expect that the surface structures and/or their temperature dependence may differ between the two materials.

An STM image of the AlN(0001) surface following growth under Al-rich conditions is shown in Fig. $1 .^{28}$ We observe a surface reconstruction consisting of an array of corrugation maxima separated by about $2.5 \mathrm{~nm}$. This result is quite different from what is observed on the $\mathrm{GaN}(0001)$ surface, which appears relatively featureless on the atomic scale due to the fast motion of the surface atoms (occasionally a $1 \times 1$ pattern is observed on that surface, which is interpreted as reflecting the $1 \times 1$ corrugation of the underlying GaN crystal). ${ }^{2}$ Surface structures identical to that of Fig. 1 have been observed for AlN films grown both on $\mathrm{GaN}$ buffer layers and grown directly on $\mathrm{SiC},{ }^{28}$ 
indicating that the surface arrangements are determined purely by $\mathrm{Al}$ atoms, i.e. not involving $\mathrm{Ga}^{29}$ A second surface reconstruction is also commonly observed on such AlN films following growth, having a corrugation period of $1.0 \mathrm{~nm}$. Both of the $2.5-\mathrm{nm}$-period and the 1.0-nm-period structures are found in LEED to have a structure that is rotated relative to the GaN $1 \times 1$, so that these to structures are identified as $2 \sqrt{3} \times 2 \sqrt{ } 3-\mathrm{R} 30^{\circ}$ and $5 \sqrt{ } 3 \times 5 \sqrt{ } 3-\mathrm{R} 30^{\circ}$, respectively. ${ }^{28}$ Another significant feature of the diffraction pattern is that the satellite peaks around the primary ones form a nearly hexagonal pattern for both $\mathrm{AlN}$ and $\mathrm{GaN}$, but in the former case this pattern is rotated relative to the primary hexagons whereas in the latter case it is not (the origin of this detailed difference between the two cases is not understood at present).

For both $\mathrm{GaN}(0001)$ and $\mathrm{AlN}(0001)$ the basic structure terminating the surface contains 2-3 ML of metal atoms. The diffraction patterns reveal a large-unit-cell or an incommensurate arrangement of the atoms on the surface. Since the atomic spacing is less for the pure metal than for the III-nitride (3.19 $\AA$ vs. $2.8 \AA$ for GaN compared to Ga, and $3.11 \AA$ vs. $2.86 \AA$ for AlN compared to $\mathrm{Al}$ ), it is thus surmised that the metal overlayers contain the $\mathrm{Ga}$ or $\mathrm{Al}$ atoms with an interatomic spacing approximately the same as in the pure metal. At room temperature the arrangements thus form the complex nearly incommensurate structures seen in the diffraction patterns, reminiscent of the incommensurate surface structures of $\mathrm{Au}$ or $\mathrm{Pt}(111)^{30}$ or of inert gases adsorbed on those surfaces. $^{31}$ At typical growth temperature the metallic overlayers are generally disordered, i.e. melted (although for $\mathrm{AlN}(0001)$ the $2 \sqrt{3} \times 2 \sqrt{3}-\mathrm{R} 30^{\circ}$ structure, appearing in RHEED as $2 \times 6$, is reported to survive even at $\left.850^{\circ} \mathrm{C}\right) .^{32}$ Nevertheless, an understanding of the lower-temperature phases is still quite useful in firmly establishing the respective metal coverage of the surfaces. The presence of the metal adlayers, during growth, has a significant impact on the growth kinetics as discussed in Section I.

Another feature of interest in Fig. 1 is that the rows of corrugation maxima tend to meander on the surface, as indicated by the dashed lines on the image showing varying angles of the corrugation rows. The result is in contrast to most STM images of surface unit cells which usually show strict translational and rotational orientation as determined by the underlying crystal structure. For AlN(0001), it appears that the large-unit-cell (nearly incommensurate) surface structure can assume varying orientations relative to the underlying AIN lattice, producing the observed variations in the row directions.

\section{Hydrogen on GaN(0001)}

On $\mathrm{GaN}(0001)$ surfaces, adlayers of various chemical species are found to displace the Ga-bilayer on the surface. In particular, hydrogen is commonly present during MBE growth, either intentionally (during RMBE) or as a background species (during PAMBE). Typical hydrogen partial pressures are on the order of $10^{-5}$ Torr in the former case and $10^{-10}$ Torr in the latter. Figure 2 shows a phase diagram derived from RHEED observations during PA-MBE growth in which some $\mathrm{H}$ has been added to the $\mathrm{N}_{2}$ flowing through the plasma source. ${ }^{33}$ The inset of Fig. 2 shows optical spectra of the plasma with and without the $\mathrm{H}$ present. In the former case sharp spectral lines at 486 and $656 \mathrm{~nm}$ are observed, arising from atomic transitions of $\mathrm{H}$, with the other features in the spectra arising from atomic and molecular transitions of $\mathrm{N}$.

In Fig. 2, the amount of $\mathrm{H}$ present is indicated by a chemical potential, $\mu_{\mathrm{H}}$, computed following Ref. [34] by assuming equilibrium of a gas of $\mathrm{H}_{2}$ molecules at the 
known partial pressure and at the growth temperature of $\approx 780^{\circ} \mathrm{C}$. Values of $\mu_{\mathrm{H}}$ thus provide a measure of the abundance of the $\mathrm{H}$ (we note that since we use a plasma to dissociate the $\mathrm{H}_{2}$ molecules our computed values of $\mu_{\mathrm{H}}$ could significantly underestimate the abundance of atomic $\mathrm{H}$, as further discussed below). Under Ga-rich conditions and at low values of $\mu_{\mathrm{H}}$ (i.e. small amounts of $\mathrm{H}$ ), the Ga-bilayer is found to persist on the surface, but the critical flux for the rough-to-smooth transition shifts to higher values of $\mathrm{Ga}$ flux. A possible interpretation for this behavior is that the $\mathrm{H}$ atoms somehow affect the gas-phase kinetics so as to produce additional active $\mathrm{N}$ species. ${ }^{33,35}$ At higher values of $\mu_{\mathrm{H}}$ a $2 \times 2$ structure forms on the surface, which is interpreted as arising from a surface termination by $\mathrm{H}$ and $\mathrm{NH}_{2}$ or $\mathrm{NH}_{3}$. The theoretical results of Van de Walle and Neugebauer $^{21}$ indicate a variety of such energetically-favorable $2 \times 2$ terminations, although occurring (for Ga-rich conditions) at values of $\mu_{\mathrm{H}}$ about $1.0 \mathrm{eV}$ higher than those of Fig. 2.

When growth is performed at the lower-values of $\mu_{\mathrm{H}}$, when the surface is still covered by the Ga-bilayer, the presence of $\mathrm{H}$ is also found to induce facetting of the surface, as illustrated in Fig. 3. Figure 3(a) shows an atomic force microscopy (AFM) image of a surface grown with $\mathrm{H}$ (at $\mu_{\mathrm{H}} \approx-1.7 \mathrm{eV}$, corresponding to about $2 \times 10^{-6}$ Torr of $\mathrm{H})$. We find large hexagonal islands on the surface, and these islands grow larger as the growth proceeds. In between the islands the terraces are still relatively flat, as shown in Fig. 3(b), similar to what is found for growth in the absence of $\mathrm{H}^{10}{ }^{10}$ Based on the AFM data the sidewalls of these islands are found to have angles of $>(45 \pm 5)^{\circ}$ relative to the (0001) plane, but convolution with the AFM tip shape prevents a more definitive determination. From $x$-ray measurements on the same samples we find that the sidewalls have lateral normal vectors of $(10 \overline{1} n),{ }^{36}$ and from the angles of $>(45 \pm 5)^{\circ}$ we thus deduce values of $n$ of 0,1 , or 2 . Scanning electron microscopy (SEM) of a particular large island is shown in Fig. 3(c). The sidewalls are clearly visible, from which we conclude that they cannot have $(10 \overline{1} 0)$ orientation (i.e. $n \neq 0)$. Tilting of the sample during SEM imaging was also performed in an effort to determine the facet angles but the results were inconclusive. We do note, however, that prior work by cross-sectional transmission electron microscopy for GaN grown in the presence of $\mathrm{H}$ reveals the presence of $(10 \overline{1} 1)$ facets, ${ }^{37}$ a result that is consistent with our measurements.

The observed formation of vicinal (10 $\overline{1} 1)$ facets on $\mathrm{GaN}$ under growth conditions in which hydrogen is abundant suggests that hydrogen termination of the facets could be the driving force for their formation. It is therefore of interest to determine if it is energetically favorable for the facets to be terminated by $\mathrm{H}$. Density functional calculations $^{38}$ together with the finite temperature thermodynamics approach ${ }^{34}$ may be utilized to determine the conditions under which the vicinal surface is clean, or terminated by $\mathrm{H}$. This approach has been employed for $\mathrm{H}$ on the $(10 \overline{1} 0)$ surface, ${ }^{34}$ the (0001) surface $^{21}$ and the (000 $\left.\overline{1}\right)$ surface $^{39}$ Recent calculations for the GaN(000 $\left.\overline{1}\right)$ surface $^{39}$ indicate a transition from a $\mathrm{H}$-terminated surface to a surface covered by a $\mathrm{Ga}$ adlayer depending on the $\mathrm{Ga}$ and $\mathrm{H}$ chemical potentials, and such a transition appears to have been observed. ${ }^{40}$ It is therefore plausible that such a transition may also occur on the GaN(10 $\overline{1} 1)$ surface.

It is clear that vicinal facets often play an important role in the growth of GaN. For example, such facets appear as the surface sidewalls of the inverted hexagonal 
pyramids that form at the termination of dislocations on the (0001) surface. The (10 $\overline{1} 1)$ surface also appears in epitaxial lateral overgrowth as a side facet. ${ }^{41}$

The use of a thermodynamics approach to determine the relative energies of the clean and H-terminated surfaces assumes that the system is in equilibrium. This assumption may not be completely valid in cases where atomic hydrogen is produced in a plasma, as discussed above. The plasma creates free $\mathrm{H}$ atoms in the gas phase that are in a highly excited state with respect to molecular $\mathrm{H}_{2}$, and the abundance of $\mathrm{H}$ in the gas phase is hence much higher than would occur simply by thermal dissociation of the molecules. However, it is well known for plasma sources of the type we use that the extraction efficiency of atoms is only about $5 \%$, with considerable recombination of the dissociated atoms occurring in the source. ${ }^{35}$ But importantly, for the present experiments, this recombination can occur with $\mathrm{N}$ thus forming $\mathrm{NH}, \mathrm{NH}_{2}$, and ammonia which themselves will act as sources of $\mathrm{H}$ at the surface. A complete description of the thermodynamics of the gas phase is beyond the scope of this work, and we will proceed simply with the use the $\mathrm{H}$ chemical potential associated with a gas of $\mathrm{H}_{2}$ molecules, ${ }^{34}$ bearing in mind the possible uncertainty of about $1.0 \mathrm{eV}$ in this quantity as discussed above in connection with the $2 \times 2$ formation. The use of a $\mathrm{H}$ chemical potential associated with an $\mathrm{H}_{2}$ gas is expected to be better justified in the case of growth by metal-organic chemical vapor deposition (MOCVD) using $\mathrm{H}_{2}$ carrier gas, where both the temperature and pressure is higher and $\mathrm{H}$ is the dominant species.

The present results are based on local density functional total energy calculations employing a plane-wave basis, a supercell geometry, and first-principles pseudopotentials. ${ }^{42,43,44,45}$ The Ga $3 \mathrm{~d}$ electrons are included in the valence bands, and the plane-wave cutoff energy is $60 \mathrm{Ry}$. Calculations for the hydrogen terminated $\mathrm{GaN}$ surfaces were performed using a $\mathrm{c}(2 \times 4)$ supercell having 8 surface atoms in each cell. An $8 \times 2$ Monkhorst-Pack k-point sampling is employed. On the vicinal $(10 \overline{1} 1)$ surface the electron counting rule is satisfied by placing $9 \mathrm{H}$ atoms on the $12 \mathrm{~N}$ dangling bonds in each unit cell. The dangling bonds arise from four $\mathrm{N}$ atoms with two dangling bonds each and four $\mathrm{N}$ atoms having a single dangling bond each. The structure is illustrated schematically in Fig. 4. The Ga adlayer structure is obtained by removing the $\mathrm{H}$ and the top layer of $\mathrm{N}$ atoms. This leads to a surface with $8 \mathrm{Ga}$ atoms per cell. Four are bonded to one $\mathrm{N}$ atom below and four are bonded to two $\mathrm{N}$ atoms below.

The energy difference between the Ga adlayer and the H-terminated surface may be written as

$$
\Delta \Omega=\Delta \mathrm{E}-8 \mu_{\mathrm{N}}-9 \mu_{\mathrm{H}}(\mathrm{p}, \mathrm{T})+\mathrm{F}_{\mathrm{H}-\mathrm{vib}}(\mathrm{T})
$$

where $\Delta \mathrm{E}=\mathrm{E}(\mathrm{H}$-terminated $)-\mathrm{E}(\mathrm{Ga}$-adlayer $)$ is the difference in the total energies calculated for the two systems. ${ }^{34}$ The $\mathrm{N}$ and Ga chemical potentials are not independent and one may employ the condition of equilibrium with bulk GaN $\left(\mu_{\mathrm{N}}=\mu_{\mathrm{GaN}(\mathrm{bulk})}-\mu_{\mathrm{Ga}}\right)$ to write $\Delta \Omega$ in terms of the Ga chemical potential. ${ }^{46}$ The hydrogen chemical potential, $\mu_{\mathrm{H}}(\mathrm{p}, \mathrm{T})$, is calculated using the expression ${ }^{34}$ for an ideal gas of $\mathrm{H}_{2}$ molecules at temperature $\mathrm{T}$ and pressure $\mathrm{p}$. The free energy of the H-related surface vibrational modes $\left(\mathrm{F}_{\mathrm{H} \text {-vib }}\right)$ is included as discussed elsewhere. ${ }^{34}$ Negative values of $\Delta \Omega$ correspond to a more stable H-terminated surface and positive values to a more stable Ga-adlayer surface. The two dimensional chemical potential space $\left(\mu_{\mathrm{Ga}}, \mu_{\mathrm{H}}\right)$ can be partitioned into the two regions 
in which the two different structures are most stable. This is shown in Fig. 5. The boundary between the two regimes depends slightly on temperature because of the explicit dependence on $\mathrm{T}$ of the vibrational free energy of the adsorbed $\mathrm{H}$. $\left(\mathrm{F}_{\mathrm{H} \text {-vib }}(1300 \mathrm{~K})\right.$ $=0.28 \mathrm{eV} / 1 \times 1 ; \quad \mathrm{F}_{\mathrm{H} \text {-vib }}(1000 \mathrm{~K})=0.33 \mathrm{eV} / 1 \times 1$. $)$ The boundaries shown correspond to temperatures of $1000 \mathrm{~K}$ (typical for MBE) and $1300 \mathrm{~K}$ (appropriate for MOCVD).

MOCVD growth at $1300 \mathrm{~K}$ and an $\mathrm{H}_{2}$ pressure of $1 \mathrm{~atm}$ corresponds to $\mu_{\mathrm{H}}$ equal to about $-1.0 \mathrm{eV}$. In this case we see that the $\mathrm{H}$-terminated surface is more stable than the $\mathrm{Ga}$ adlayer surface for essentially the entire range of $\mathrm{Ga}$ chemical potentials.

MBE growth at $1000 \mathrm{~K}$ with a $\mathrm{H}$ partial pressure of $10^{-10}$ atm corresponds to hydrogen chemical potential of $-1.72 \mathrm{eV}$. In this case the H-terminated surface is stable only in the extreme $\mathrm{N}$-rich limit, specifically for $\mu_{\mathrm{Ga}}<\mu_{\mathrm{Ga}(\mathrm{bulk})}-0.87 \mathrm{eV}$. One must keep in mind, however, the limitation of this approach to the situation where one has equilibrium between the surface phase and the $\mathrm{H}_{2}$ gas at the specified temperature and pressure. In the present case, where most of the $\mathrm{H}_{2}$ molecules introduced into the system have been dissociated (and possibly combined with $\mathrm{N}$ ) in the plasma, the system may be far from equilibrium. It may then be more appropriate to assign to hydrogen a quasichemical potential that is much higher than what one would expect based on the ideal gas calculation. Assuming a chemical potential for hydrogen that is higher than the ideal gas value by $\sim 1 \mathrm{eV}$, as discussed above, leads to the conclusion that the vicinal surface would be terminated by hydrogen in Ga-rich conditions as well as in N-rich conditions.

\section{GaN (10 $\overline{1} 0)$}

Growth of nitride has been studied on faces other than the (0001) and (000 $\overline{1})$ c-planes, including the $(10 \overline{1} 0)$ m-plane ${ }^{47,48,49}$ and the $(11 \overline{2} 0)$ a-plane, ${ }^{50}$ with one important motivation being that some of these other directions are not polarizable i.e. no built-in (pyroelectric) or piezoelectric fields occur for those directions. ${ }^{47}$ Metallic bilayers are also found to occur on the $(10 \overline{1} 0)$ surface. STM data for a $(10 \overline{1} 0)$ oriented GaN film, grown by PA-MBE on $\mathrm{ZnO}(10 \overline{1} 0)$, are shown in Fig. $6{ }^{48}$ The overall film morphology displays $(10 \overline{1} 0)$ terraces extending as strips in the [11 $\overline{2} 0]$ direction, as shown in Fig. 6(a). For growth under Ga-rich conditions the primary reconstruction observed is indicated in Fig. 6(b), with the unit cell marked on the image. A $1 \times 1$ unit cell of the $(10 \overline{1} 0)$ surface has basis vectors of $\mathbf{a}$ in the [11 20 ] direction with length $3.19 \AA$ and $\mathbf{c}$ in the [0001] direction with length $5.19 \AA$. Within experimental uncertainty $(+10 \%)$ our observed surface unit cell has primitive translation vectors $4 \mathbf{a}+1 \mathbf{c}$ and $-1 \mathbf{a}+5 \mathbf{c}$. This structure is thus somewhat complex, but this behavior is consistent with the presence of a nearly incommensurate metallic layer on the surface, as discussed in Sections I and II for the (0001) surfaces of GaN and AlN. The RHEED diffraction patterns for this structure are shown in Figs. 6(c) and (d). An approximate 4x spacing for the electron beam along the [0001] direction is found, which is at least qualitatively consistent with the nearly $4 \times$ spacing seen for the $\left[\begin{array}{ll}11 & 2\end{array} 0\right]$ direction in Fig. 6(b) (the streaks in Fig. 6(c) clearly do not have exact 4 spacing; this behavior may arise from the rather complicated surface superstructure). For the [11 $\overline{2} 0]$ azimuth no higher-order RHEED streaks are observed, with this result being consistent with the real-space images since the surface terraces are quite limited in extent in the [0001] direction. 
The surface coverage of Ga was not determined in the above experiments, but theoretically it was found that, for Ga-rich conditions, two (or perhaps more) ML of Ga form energetically favorable surface structures. Recent work by Brandt et al. has determined a Ga coverage of $3 \mathrm{ML}$ on the $(10 \overline{1} 0)$ surface under Ga-rich conditions. ${ }^{49}$ In addition, those workers find clear $4 \times$ streaks in the RHEED patterns for both the [0001] and $[11 \overline{2} 0]$ azimuths. Those RHEED results are somewhat different than our results of Fig. 6; for the [0001] azimuth the difference might arise from the limited spatial extent of

our surface terraces. For the [11 $\overline{2} 0]$ azimuth however, the RHEED patterns appear to indicate a real difference in structure between our surface and those of Brandt et al. We do note that small differences in lattice parameter (i.e. strain) could possibly affect the structure, and such differences could occur because of the different substrates ( $\mathrm{ZnO}$ vs. $\gamma$ $\mathrm{LiAlO}_{2}$ ) used in the works. Further study is needed to resolve this discrepancy in the results and to more fully determine a complete surface structure.

\section{Summary}

In summary, considerable progress has been made in the last decade towards the understanding of the structures on GaN and AlN surfaces. Under metal-rich conditions, various crystal faces of both materials are found to be terminated by 2-3 ML of metal atoms. At room temperature these metallic layers form nearly incommensurate structures on the surfaces because of the reduced lattice parameter of the metal film compared to the III-nitride. At growth temperatures the metal films are melted, and the resulting fluid bilayer on the surface plays an important role in growth kinetics. Adlayers of other atoms on the surface can displace the metal-bilayer, and in certain cases may also provide a similar surfactant type behavior as found for the bilayer.

\section{Acknowledgements}

This work was supported by the U.S. Office of Naval Research through contracts N00014-02-0933 monitored by C. Wood, and N00014-02-0433 monitored by L. Cooper and C. Baatar. 


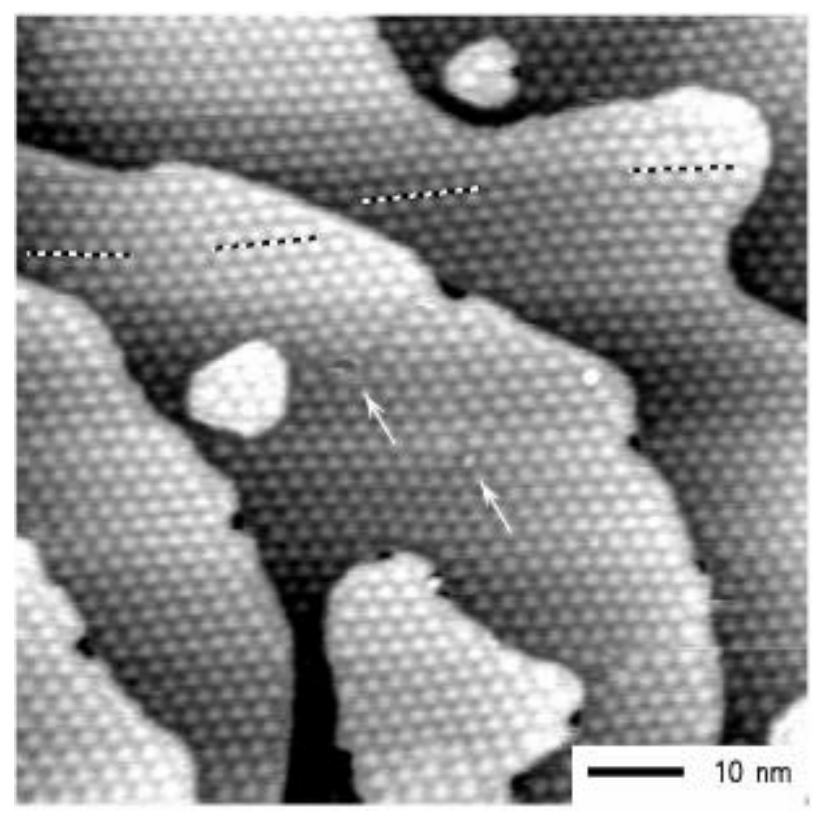

Fig. 1. STM image of an Al-rich $\mathrm{AlN}(0001)$ surface, displaying the $5 \sqrt{3} \times 5 \sqrt{3}-R 30^{\circ}$ reconstruction. The image was acquired with a sample voltage of $+1.5 \mathrm{~V}$ and is displayed with a gray scale range of $3.5 \AA$. Some distortions in the hexagonal stacking sequence are marked by dashed lines, and the arrows mark surface dislocations.

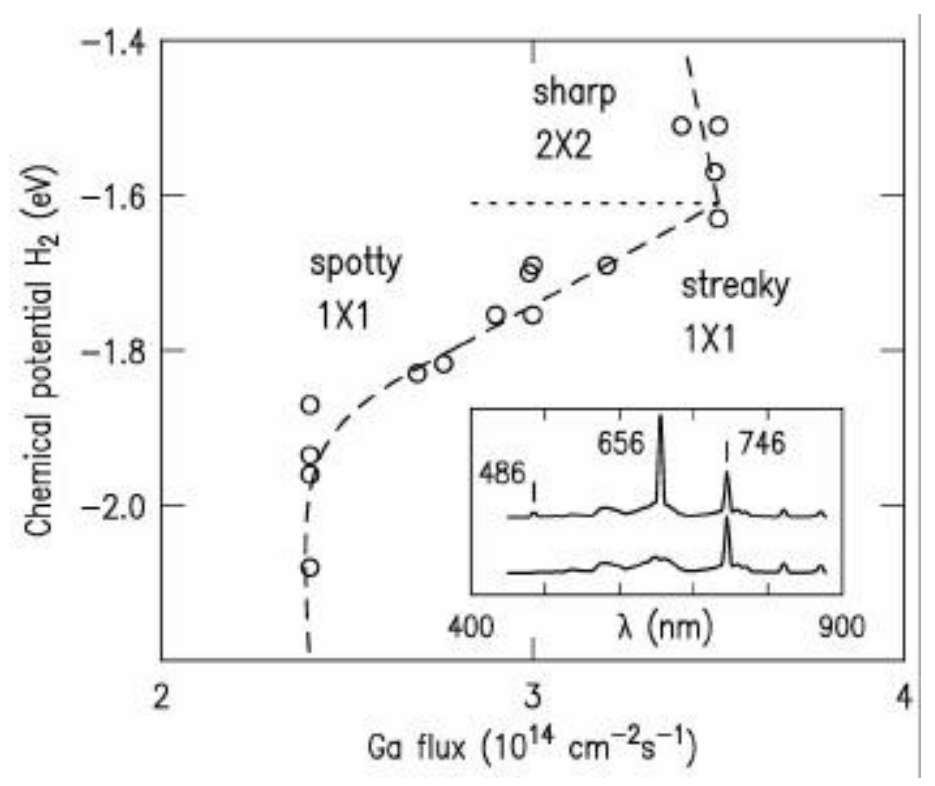

Fig. 2. Dependence of the streaky-to-spotty Ga flux transition point (circles) on the chemical potential of hydrogen. The dashed line is drawn as a guide to the eye. The dotted line is an illustrative boundary between the spotty $1 \times 1$ and sharp $2 \times 2$ phases. (Exact measurement of this line is not possible since the sharp $2 \times 2$ becomes spotty after some time). The inset shows optical spectra of the plasma emission, with (upper line) and without (lower line) the presence of $\mathrm{H}$. The wavelength (in $\mathrm{nm}$ ) of some emission lines are marked. 


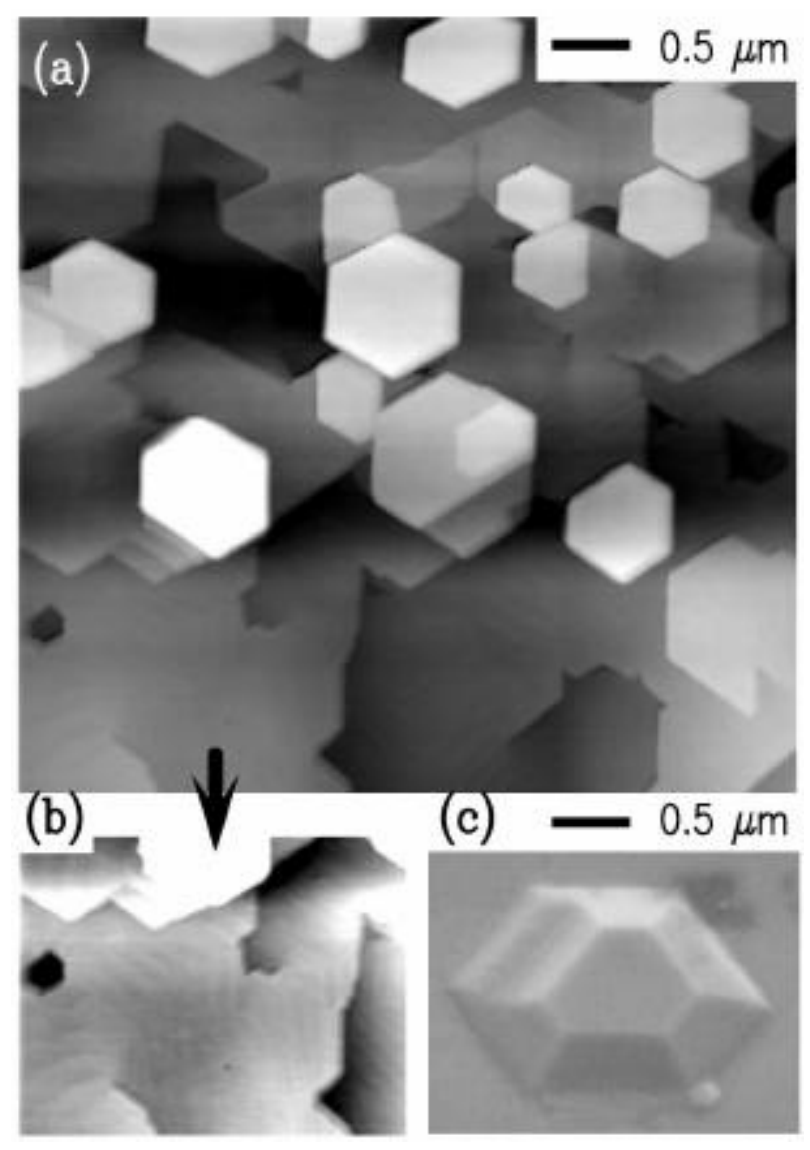

Fig. 3. AFM image of GaN film grown in the presence of $\mathrm{H}$, displayed with a gray scale range of $41 \mathrm{~nm}$. A magnified view of the lower left corner of the image is shown in (b), with a gray scale range of $13 \mathrm{~nm}$. (c) Scanning electron micrograph of a large hexagonal island on the surface. 
GaN(10-11) with 3/4 ML H

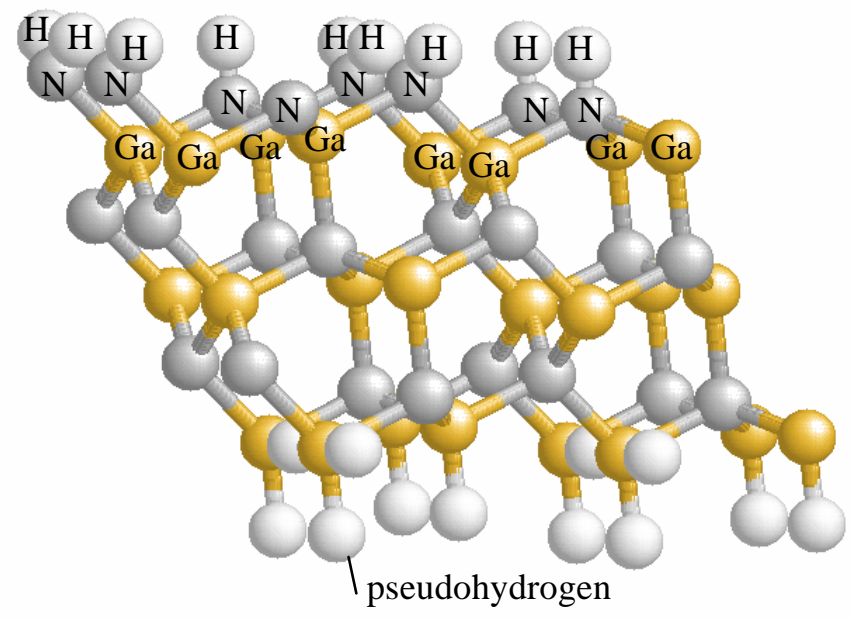

Fig. 4. (color for on-line version) A ball and stick model of the H-terminated GaN(10 11 ) surface. In this structure there are $9 \mathrm{H}$ atoms in each unit cell. There are two $\mathrm{N}-\mathrm{H}_{2}$ groups and five $\mathrm{N}-\mathrm{H}$ groups. There are three $\mathrm{N}$ dangling bonds in each cell. The Ga adlayer surface is obtained by removal of the $9 \mathrm{H}$ atoms and the $8 \mathrm{~N}$ atoms in the top layer. Four of these eight $\mathrm{Ga}$ atoms are bonded to two $\mathrm{N}$ atoms and four are bonded to a single $\mathrm{N}$ atom. The Ga atoms on the back-side of the slab are passivated by pseudohydrogen atoms having charge $5 / 4$.

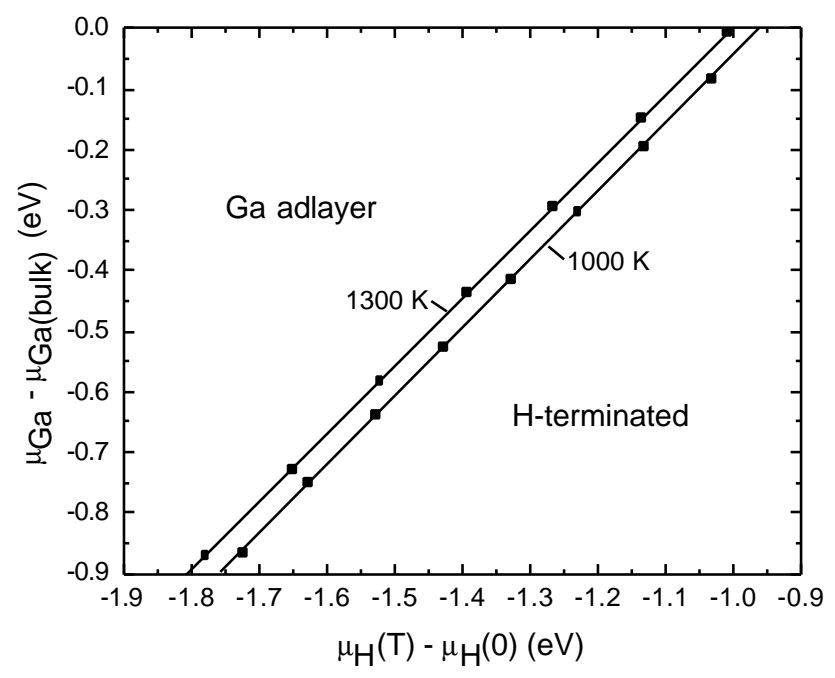

Fig. 5. This diagram illustrates the regions in chemical potential space $\left(\mu_{\mathrm{H}}, \mu_{\mathrm{Ga}}\right)$ where the $\mathrm{Ga}$ adlayer or the H-terminated structure (shown in Fig 4) are stable. Two boundaries are shown, one for a temperature of $1300 \mathrm{~K}$ and one for a temperature of $1000 \mathrm{~K}$. The explicit T-dependence arises from the vibrational free-energy term in the formation energy. For points above the lines the Ga adlayer is preferred. The Ga chemical potential may vary between the Ga-rich limit $\left(\mu_{\mathrm{Ga}}-\mu_{\mathrm{Ga}(\text { bulk })}=0.0\right)$ and the N-rich limit $\left(\mu_{\mathrm{Ga}}-\right.$ $\left.\mu_{\mathrm{Ga}(\mathrm{bulk})}=-0.9 \mathrm{eV}\right)$. Typical MOCVD growth conditions correspond to $\mu_{\mathrm{H}}-\mu_{\mathrm{H}}(\mathrm{T}=0)=$ $-1.0 \mathrm{eV}$. 

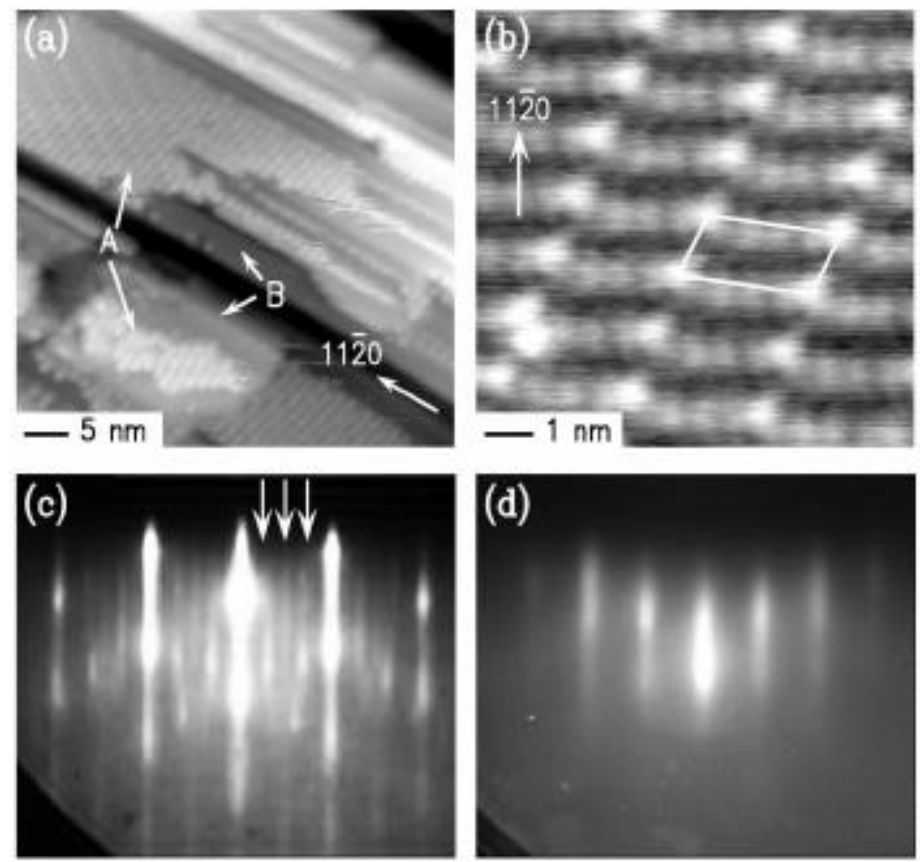

Fig. 6. (a) STM image of Ga-rich $\mathrm{GaN}(10$ 10) surface, acquired with a sample voltage of $-3.0 \mathrm{~V}$ and displayed with a gray scale range of $8 \AA$. Surface areas of different reconstructions are marked 'A' and 'B'. (b) Expanded view of the A-type surface reconstruction, acquired with a sample voltage of $-0.3 \mathrm{~V}$ and displayed with a gray scale range of $1.0 \AA$. (c) and (d) RHEED patterns for this surface, with the electron beam along the [0001] and [11 20$]$ directions, respectively. The arrows in (c) indicate a $4 \times$ spacing (the observed streaks do not precisely follow this spacing).

${ }^{1}$ R. M. Feenstra, J. E. Northrup, and J. Neugebauer, MRS Internet J. Nitride Semicond. Res. 7, 3 (2002).

2 A. R. Smith, R. M. Feenstra, D. W. Greve, M.-S. Shin, M. Skowronski, J. Neugebauer, and J. Northrup, J. Vac. Sci. Technol B 16, 2242 (1998).

${ }^{3}$ C. Adelmann, J. Brault, G. Mula, B. Daudin, L. Lymperakis, and J. Neugebauer, Phys. Rev. B 67, 165419 (2003).

${ }^{4}$ G. Koblmüller, R. Averbeck, H. Riechert, and P. Pongratz, Phys. Rev. B 69, 035325 (2004).

${ }^{5}$ A. Pavlovska, E. Bauer, V. M. Torres, J. L. Edwards, R. B. Doak, I. S. T. Tong, V. Ramachandran, and R. M. Feenstra, J. Cryst. Growth 189/190, 310 (1998).

${ }^{6}$ A. R. Smith, R. M. Feenstra, D. W. Greve, M.-S. Shin, M. Skowronski, J. Neugebauer, and J. Northrup, Surf. Sci. 423, 70 (1999).

${ }^{7}$ J. E. Northrup, J. Neugebauer, R. M. Feenstra, A. R. Smith, Phys. Rev. B 61, 9932 (2000).

${ }^{8}$ S. H. Xu, H. Wu, X. Q. Dai, W. P. Lau, L. X. Zheng, M. H. Xie, and S. Y Tong, Phys. Rev. B 67, 125409 (2003). 
${ }^{9}$ B. Heying, R. Averbeck, L. F. Chen, E. Haus, H. Riechert, and J. S. Speck, J. Appl. Phys. 88, 1855 (2000).

${ }^{10}$ C. D. Lee, V. Ramachandran, Ashutosh Sagar, R. M. Feenstra, D. W. Greve, W. L. Sarney, L. Salamanca-Riba, D. C. Look, Song Bai, W. J. Choyke, and R. P. Devaty, J. Electron. Mater. 30, 162 (2001).

${ }^{11}$ K. H. Ploog, O. Brandt, R. Muralidharan, A. Thamm, and P. Waltereit, J. Vac. Sci. Technol. B 18, 2290 (2000).

12 J. Neugebauer, T. K. Zywietz, M. Scheffler, J. E. Northrup, H. Chen, and R. M. Feenstra, Phys. Rev. Lett. 90, 056101 (2003).

${ }^{13}$ G. Muda, C. Adelmann, S. Moehl, J. Oullier, and B. Daudin, Phys. Rev. B 64, 195406 (2001).

${ }^{14}$ C. Adelmann, J. Brault, D. Jalabert, P. Gentile, H. Mariette, G. Mula, and B. Daudin, J. Appl. Phys. 91, 9638 (2002).

${ }^{15}$ J. W. P. Hsu, M. J. Manfra, D. V. Lang, S. Richter, S. N. G. Chu, A. M. Sergent, R. N. Kleiman, L. N. Pfeiffer, and R. J. Molnar, Appl. Phys. Lett. 78, 1685 (2001).

${ }^{16}$ J. W. P. Hsu, M. J. Manfra, S. N. G. Chu, C. H. Chen, L. N. Pfeiffer, and R. J. Molnar, Appl. Phys. Lett. 78, 3980 (2001).

${ }^{17}$ J. E. Northrup, Phys. Rev. B 66, 045204 (2002).

${ }^{18}$ N. Grandjean, M. Leroux, J. Massies, M. Mesrine, and M. Laügt, Jpn. J. Appl. Phys. 38, 618 (1999).

${ }^{19}$ A. Thamm, O. Brandt, Y. Takemura, A. Trampert, and K. H. Ploog, Appl. Phys. Lett. 75, 944 (1999).

${ }^{20}$ S. Vézian, F. Semond, J. Massies, D. W. Bullock, Z. Ding, and P. M. Thibado, Surf. Sci. 541, 242 (2003).

${ }^{21}$ C. Van de Walle and J. Neugebauer, Phys. Rev. Lett. 88, 066103 (2002).

${ }^{22}$ F. Widmann, B. Daudin, G. Feuillet, N. Pelekanos, and J. L. Rouvière, Appl. Phys. Lett. 73, 2642 (1998).

${ }^{23}$ H. Chen, R. M. Feenstra, J. E. Northrup, T. Zywietz, J. Neugebauer, and D. W. Greve, J. Vac. Sci. Technol. B 18, 2284 (2000).

${ }^{24}$ V. Ramachandran, R. M. Feenstra, J. E. Northrup, and D. W. Greve, MRS Internet J. Nitride Semicond. Res. 5S1, W3.65 (2000).

${ }^{25}$ E. Monroy, T. Andreev, P. Hollinger, E. Bellet-Amalric, T. Shibata, M. Tanaka, and B. Daudin, Appl. Phys. Lett. 84, 2554 (2004).

${ }^{26}$ V. Ramachandran, C. D. Lee, R. M. Feenstra, A. R. Smith, J. E. Northrup, and D. W. Greve, J. Cryst. Growth 209, 355 (2000).

${ }^{27}$ J. Neugebauer, Phys. Stat. Sol. (c) 0, 1651 (2003).

${ }^{28}$ C. D. Lee, Y. Dong, R. M. Feenstra, J. Northrup, and J. Neugebauer, Phys. Rev. B 68, 205317 (2003).

${ }^{29}$ Additionally, regarding the use of GaN buffer layers, Auger electron spectroscopy performed on the AlN surfaces revealed no detectable Ga so long as the GaN buffer layers were grown without $\mathrm{Ga}$ droplets.

${ }^{30}$ A. R. Sandy, S. G. J. Mochrie, D. M. Zehner, G. Grübel, K. G. Huang, and D. Gibbs, Phys. Rev. Lett. 68, 2192 (1992); D. L. Abernathy, D. Gibbs, G. Grübel, K. G. Huang, S. G. J. Mochrie, A. R. Sandy, and D. M. Zehner, Surf. Sci 283, 260 (1993); U. Harten, A. M. Lahee, J. P. Toennies, and Ch. Wöll, Phys. Rev. Lett. 54, 2619 (1985). 
${ }^{31}$ See e.g. J. F. Zhu, H. Ellmer, H. Malissa, T. Brandstetter, D. Semrad, and P. Zeppenfeld, Phys. Rev. B 68, 045406 (2003).

${ }^{32}$ H. P. D. Schenk, G. D. Kipshidze, U. Kaiser, A. Fissel, J. Käusslich, J. Schulze, and W. Richter, J. Cryst. Growth 200, 45 (1999).

${ }^{33}$ Y. Dong and R. M. Feenstra, Phys. Stat. Sol. (c), to appear.

${ }^{34}$ J. E. Northrup, R. Di Felice, and J. Neugebauer, Phys. Rev. B 56, 4325 (1997).

${ }^{35}$ B. L. VanMil, H. Gue, L. J. Holbert, K. Lee, T. H. Myers, T. Liu, and D. Korakakis, J. Vac. Sci. Technol. B 22, 2149 (2004) and references therein.

${ }^{36}$ Y. Dong, R. M. Feenstra, D. W. Greve, and J. C. Moore, M. D. Sievert, and A. A. Baski, submitted to Appl. Phys. Lett.

${ }^{37}$ T. Araki, A. Onogi, N. Juni, and Y. Nanishi, J. Cryst. Growth, 237, 983 (2002).

${ }^{38}$ W. Kohn and L. J. Sham, Phys. Rev. 140, 1133 (1965).

39 J. E. Northrup and J. Neugebauer, Appl. Phys. Lett. 85, 3429 (2004).

${ }^{40}$ R. Held, D. E. Crawford, A. M. Johnston, A. M. Dabiran, and P. I. Cohen, Surface Review and Letters 5, 913 (1998).

${ }^{41}$ O.-H. Nam, M. D. Bremser, T. S. Zheleva, and R. F. Davis, Appl. Phys. Lett. 71, 2638 (1997).

${ }^{42}$ R. Stumpf and M. Scheffler, Comp. Phys. Comm. 79, 447 (1994).

${ }^{43}$ N. Troullier and J. L. Martins, Phys. Rev. B 43, 1993 (1991).

${ }^{44}$ J. P. Perdew and A. Zunger, Phys. Rev. B 23, 5048 (1981).

${ }^{45}$ H. Monkhorst and J. Pack, Phys. Rev. B 13, 5188 (1976).

${ }^{46}$ G. -X. Qian, R. M. Martin, and D. J. Chadi, Phys. Rev. B 38, 7649 (1988).

${ }^{47}$ P. Waltereit, O. Brandt, M. Ramsteiner, A. Trampert, H. T. Grahn, J. Menniger, M. Reiche, R. Uecker, P. Reiche, and K. H. Ploog, Phys. Stat. Sol. (a) 180, 133 (2000).

${ }^{48}$ C. D. Lee, R. M. Feenstra, J. E. Northrup, L. Lymperakis, and J. Neugebauer, Appl. Phys. Lett. 82, 1793 (2003).

${ }^{49}$ O. Brandt, Y. Sun, L. Däweritz, and K. H. Ploog, Phys. Rev. B 69, 165326 (2004).

${ }^{50}$ M. McLaurin, B. Haskell, S. Nakamura, and J. S. Speck, J. Appl. Phys. 96, 327 (2004). 